\title{
Neutron-induced capture cross sections of short-lived actinides with the surrogate reaction method
}

\author{
M. Aïche ${ }^{1, a}$, G. Boutoux ${ }^{1}$, B. Jurado ${ }^{1}$, G. Barreau ${ }^{1}$, L. Matthieu ${ }^{1}$, S. Czajkowski ${ }^{1}$, D. Dassie ${ }^{1}$, B. Haas ${ }^{11}$, V. Méot $^{2}$, \\ O. Roig ${ }^{2}$, L. Gaudefroy ${ }^{2}$, J.Taieb ${ }^{2}$, N. Pillet ${ }^{2}$, T. Faul ${ }^{2}$, O. Sérot ${ }^{3}$, E. Bauge ${ }^{2}$, and F. Gunsing ${ }^{4}$ \\ 1 CENBG, Chemin du Solarium B.P. 120, 33175 Gradignan, France \\ 2 CEA DAM DIF, F-91297 Arpajon, France \\ 3 CEA-Cadarache, DEN/DER/SPRC/LEPh, 13108 Saint Paul lez Durance, France \\ 4 CEA Saclay, DSM/DAPNIA/SPhN, 91191 Gif-sur-Yvette cedex, France
}

\begin{abstract}
Determination of neutron-capture cross sections of short-lived nuclei is opening the way to understand and clarify the properties of many nuclei of interest for nuclear structure physics, nuclear astrophysics and particularly for transmutation of nuclear wastes. The surrogate approach is well-recognized as a potentially very useful method to extract neutron cross sections for low-energy compound-nuclear reactions and to overcome the difficulties related to the target radioactivity. In this work we will assess where we stand on these neutron-capture cross section measurements and how we can achieve the short-lived Minor Actinides nuclei involved in the nuclear fuel cycle. The CENBG collaboration applied the surrogate method to determine the neutron-capture cross section of ${ }^{233} \mathrm{~Pa}\left(T_{1 / 2}=27 \mathrm{~d}\right)$. The ${ }^{233} \mathrm{~Pa}(\mathrm{n}, \gamma)$ cross section is then deduced from the measured gamma decay probability of ${ }^{234} \mathrm{~Pa}$ compound nucleus formed via the surrogate ${ }^{232} \mathrm{Th}\left({ }^{3} \mathrm{He}, \mathrm{p}\right)$ reaction channel. The obtained cross section data, covering the neutron energy range 0.1 to $1 \mathrm{MeV}$, have been compared with the predictions of the Hauser-Feshbach statistical model. The importance of establishing benchmarks is stressed for the minor actinides region. However, the lack of desired targets led us to propose recently the ${ }^{174} \mathrm{Yb}\left({ }^{3} \mathrm{He}, \mathrm{p} \gamma\right)$ reaction as a surrogate reaction for the $(\mathrm{n}, \gamma)$ predetermined benchmark cross section of ${ }^{175} \mathrm{Lu}$. An overview of the experimental setup combining gamma ray detectors such as Ge and C6D6 in coincidence with light charged particles $\triangle \mathrm{E}-\mathrm{E}$ Telescopes will be presented and preliminary results will be discussed.
\end{abstract}

\section{Introduction}

Neutron-induced data of short-lived nuclei are of great interest in applied science and fundamental physics. The CENBG collaboration measures fission and capture data of interest for the development of the Th fuel cycle and for minor actinides incineration. However, such cross sections are very difficult to measure directly due to the background contributions and fabrication issues on account of the radioactive decay of the targets. These difficulties can be overcome with the so-called surrogate reaction technique, developed in the 1970's by Cramer and Britt Ref [1]. It consists in measuring the decay probability of a compound nucleus (e.g., fission, neutron emission, or radiative capture) produced via an alternative (surrogate) reaction, in this case we use a few-nucleon transfer reaction, as illustrated in figure 1. The left part of figure 1 illustrates a "direct" neutron-induced reaction on the target A-1, which leads to the compound-nucleus $\mathrm{A}$ at an excitation energy $\mathrm{E}^{*}$. The nucleus A can decay through different exit channels: fission, $\gamma$, neutron emission, etc... On the right part of figure 1 , in the surrogate reaction method, the same compound nucleus $\mathrm{A}$ is produced by a transfer reaction between a projectile y (a light charged particle) and a target X. In the

\footnotetext{
a e-mail: aiche@cenbg.in2p3.fr
}

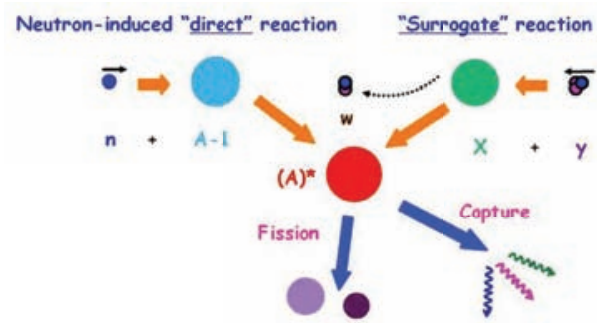

Fig. 1. Schematic representation of the surrogate reaction method. The surrogate reaction is here a transfer reaction $\mathrm{X}(\mathrm{y}, \mathrm{w}) \mathrm{A}^{*}$. Two possible exit channels, fission and capture, are also represented

context of short-lived nuclei, the interest of this method is to use targets which are less radioactive than the target A1. The transfer reaction $(y+X \rightarrow A+w)$ leads to a heavy recoil nucleus $\mathrm{A}$ and an outgoing particle $\mathrm{w}$ (proton, deuton, triton, etc). The identification of the ejectile $w$ permits to determine the mass $\mathrm{A}$ and charge $\mathrm{Z}$ of the decaying nucleus. In addition, we can deduce the excitation energy $\mathrm{E}^{*}$ of the compound nucleus A by measuring the kinetic energy and the emission angle of the ejectile w. Experimentally, the detection of the ejectile w, in coincidence or not,

This is an Open Access article distributed under the terms of the Creative Commons Attribution-Noncommercial License, which permits unrestricted use, distribution, and reproduction in any noncommercial medium, provided the original work is properly cited. 
with a given decay product (fission fragments or gamma rays) of the compound nucleus $A^{*}$ gives the decay probability $P_{\text {decay }}^{A, \exp }\left(E^{*}\right)$ of the given reaction channel. According to the surrogate reaction method, this measurement of the fission or capture probability permits to determine the neutron-induced cross section for the nucleus A-1 according to the equation:

$$
\sigma_{\text {decay }}^{A-1}\left(E_{n}\right)=\sigma_{C N}^{A}\left(E_{n}\right) \cdot P_{\text {decay }}^{A, \exp }\left(E^{*}\right)
$$

where $\sigma_{C N}^{A}\left(E_{n}\right)$ is the compound nuclear formation cross section in the desired reaction (formation of the nucleus A after a neutron absorption with an energy En). In our case, $\sigma_{C N}^{A}\left(E_{n}\right)$ is obtained from the optical model calculations developed in CEA - DIF Bruyères-le-Chatel. However, the average angular momentum of the populated states by a transfer reaction are not the same as those populated in a neutron-induced reaction. The issue is that fission or capture probabilities depend on the quantum states $(\mathrm{J}, \boldsymbol{\pi})$ of the compound nucleus. More exactly, the neutron-induced cross sections of the nucleus A-1 should be written:

$$
\sigma_{\text {decay }}^{A-1}\left(E_{n}\right)=\sum_{J, \pi} \sigma_{N . C}^{A}\left(E_{n}, J, \pi\right) \cdot G_{\text {decay }}^{A}\left(E^{*}, J, \pi\right)
$$

where $\mathrm{G}$ are the branching ratios for a given decay channel. Actually, the surrogate reaction method could be applied only if the decay probabilities from neutron-induced and transfer reactions are similar:

$$
P_{\text {neutron,decay }}=P_{\text {transfer,decay }}
$$

In the Hauser-Feshbach formulation Ref [2], these decay probabilities are given by:

$$
\begin{gathered}
P_{\text {neutron }}^{\text {decay }}=\sum_{J, \pi} P_{\text {neutron }}^{\text {form }}\left(E^{*}, J, \pi\right) \cdot G^{\text {decay }}\left(E^{*}, J, \pi\right) \\
P_{\text {transfer }}^{\text {decay }}=\sum_{J, \pi} P_{\text {transfer }}^{\text {form }}\left(E^{*}, J, \pi\right) \cdot G^{\text {decay }}\left(E^{*}, J, \pi\right)
\end{gathered}
$$

where $P_{\text {neutron }}^{\text {form }}\left(E^{*}, J, \pi\right)$ corresponds to the probability that the compound nucleus is formed in the desired reaction with spin $\mathrm{J}$ and parity $\pi$, and subsequently decayed with the branching ratio $G^{\text {decay }}\left(E^{*}, J, \pi\right)$. Equation (3) may be valid in two cases: First hypothesis : The decay probabilities of the compound nucleus are independent of $\mathrm{J}$ and $\pi$. By writing,

$$
G^{\text {decay }}\left(E^{*}, J, \pi\right)=G^{\text {decay }}\left(E^{*}\right)
$$

we denote the $\mathrm{J} \pi$-independent decay probability for the exit channel and they can so be taken out of the summation signs in equation (4) and (5). It may be noted here that $\sum_{J, \pi} P_{\text {neutron }}^{\text {form }}\left(E^{*}, J, \pi\right)=1$ and the cross section for the desired reaction takes the simple product form of the equation (2). This hypothesis is known as the WeisskopfEwing approximation and is justified for excitation energies where the decay of the compound-nucleus is dominated by statistical level densities. The conditions under which the Weisskopf-Ewing limit applies have been investigated in Ref [3]. Almost all applications of the surrogate method have made use of this approximation. However, for low excitation energy $\mathrm{E}^{*}$, the decay probabilities strongly depend of the spin of discrete states, whose population depends on the reaction mechanism used to produce the nucleus A. Second hypothesis: For lower excitation energy $\mathrm{E}^{*}$, eq. (3) is valid only if the $\mathrm{J} \pi$ distributions populated in the two reactions are similar:

$$
P_{\text {transfer }}^{f o r m}\left(E^{*}, J, \pi\right) \approx P_{\text {neutron }}^{f o r m}\left(E^{*}, J, \pi\right)
$$

Unfortunately, angular momentum populations are still poorly known experimentally and theoretically. An important effort from theoreticians and experimentalists should be done in order to determine these distributions. The use of the Weisskopf-Ewing approximation in the analysis of Surrogate experiments is typically justified a posteriori by comparing the extracted cross sections to the existing "direct" measurements. In this contribution, we focus on capture cross section measurements which, compared to fission, may be more sensitive to the difference of spin-parity distributions populated in neutron-induced and surrogate reactions. Moreover, in the case of minor actinides, the competition with fission process involves low exitation energy measurements, where the decay widths are not dominated by the statistical level density. Note also that the WeisskopfEwing approximation should not be valid when the angular momentum of the $\mathrm{CN}$ is not much larger than the spincutoff parameter of the level density distribution, which, for the actinide region, is about $7 \hbar$.

\section{Capture cross sections for $233 \mathrm{~Pa}$}

In the first $(\mathrm{n}, \gamma)$ surrogate experiment of CENBG collaboration, the surrogate reaction ${ }^{232} \mathrm{Th}\left({ }^{3} \mathrm{He}, \mathrm{p}\right) 234 \mathrm{~Pa}$ was used to obtain the capture cross section of ${ }^{233} \mathrm{~Pa}$. This nucleus plays a very important role in the Th cycle and has a half life of 27 days.

The $\gamma$-ray emission probability distribution of ${ }^{233} \mathrm{~Pa}(n, \gamma)^{*}$ has been measured between 5.2 and $6.2 \mathrm{MeV}$ excitation energy using the previous transfer reaction at an incident $3 \mathrm{He}$ energy of $24 \mathrm{MeV}$ and current of 20 particle nA. The experimental set-up is made of four liquid scintillator C6D6 detectors and four standard Si telescopes $(\triangle \mathrm{E} / \mathrm{E})$ arranged respectively to detect $\gamma$ rays and charged particles in coincidence. In order to determine the total number of $\gamma$-ray cascades in a given nucleus, the raw experimental detected gamma-ray events have to be corrected by the weighting function technique. This weighting requires complete knowledge of the efficiency and response functions for the C6D6 detector set-up and surroundings. These have been determined experimentally using gamma-ray sources, inelastic scattering reactions and transfer reactions on light nuclei. The collected data divide into two groups, namely (i) the energy spectra of the protons in singles, $N_{\text {single }}(E *)$ and (ii) the energy spectra of the protons in coincidence with gamma rays detected by at least one of the C6D6 scintillators, $N_{\text {coinc }}(E *), \mathrm{E}^{*}$ being the excitation energy of $234 \mathrm{~Pa}$. 


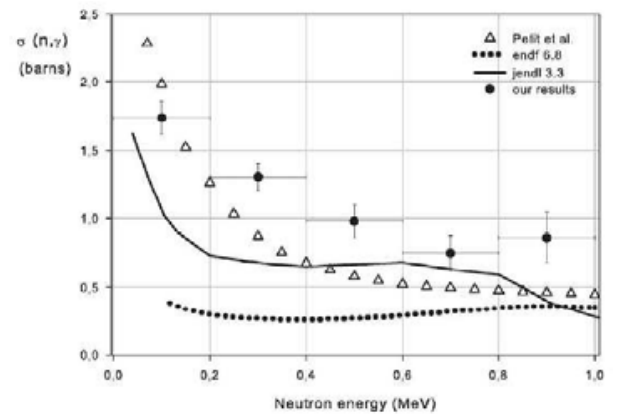

Fig. 2. Radiative capture cross section of $233 \mathrm{~Pa}$ compared to various predictions

The experimental radiative capture probability $P_{(n, \gamma)}$ can be defined as:

$$
P_{(n, \gamma)}(E *)=1 / \epsilon_{w}(E *) * N_{\text {coinc }}(E *) / N_{\text {single }}(E *)
$$

where $\epsilon_{w}$ represents the weighted photon detection efficiency.

Under the Weisskopf-Ewing assumption that the population for the intermediate states is the same for both the ${ }^{232} \mathrm{Th}\left({ }^{3} \mathrm{He}, p\right)$ and ${ }^{233} \mathrm{~Pa}(n, \gamma)$, the $(\mathrm{n}, \gamma)$ capture cross section of $233 \mathrm{~Pa}$, in the neutron energy range 0 to $1 \mathrm{MeV}$, has been deduced via the product of the measured $\gamma$ emission probability of $234 \mathrm{~Pa}^{*}$ with the calculated compound nucleus formation cross section of the reaction $233 \mathrm{~Pa}+$ n. It was not possible to extend this window to higher energy because fission becomes a competing process above $1 \mathrm{MeV}$ (see Ref [7]) and consequently gamma rays from the fission fragments will contaminate the $\gamma$ spectra. Our results are presented in 2 together with statistical model calculations (using fission barrier parameters) of Petit et al. Ref [4] and recommended values extracted from the evaluated nuclear data files JENDL-3 and ENDF/B-VI. It should however be pointed out that these surrogate measurements could not be validated for the presently investigated gamma decay channel because the $233 \mathrm{~Pa}(\mathrm{n}, \gamma)$ experimental direct capture cross sections for low energy neutrons are not known.

\section{Capture cross sections for $175 \mathrm{Lu}$}

The production of evaporated metallic isotopically-pure actinide targets is extremely complicated. Therefore as a first step we have chosen to validate the surrogate method for capture reactions on rare earth nuclei. Our aim is to study the transfer reaction $174 \mathrm{Yb}(3 \mathrm{He}, \mathrm{p} \gamma) 176 \mathrm{Lu}$ as surrogate for the $175 \mathrm{Lu}(\mathrm{n}, \gamma)$ cross sections. We have considered the $175 \mathrm{Lu}(\mathrm{n}, \gamma)$ cross section because it presents the advantage to be one of the best known capture cross sections, see for example Ref [8] and [9]. Moreover, a program has been developed using the $4 \pi$ DANCE detector array operated in the Lujan Center at the Los Alamos National Laboratory to expand the available databases for $175,176 \mathrm{Lu}(\mathrm{n}, \gamma)$ in the neutron energy range $[\mathrm{meV}, \mathrm{keV}]$. Then the $175 \mathrm{Lu}(\mathrm{n}, \gamma)$

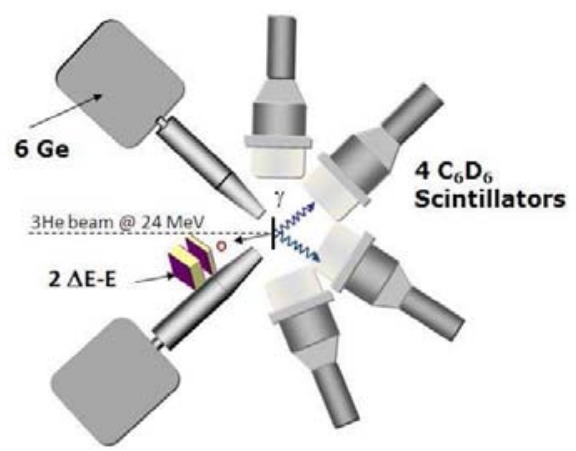

Fig. 3. Experimental set-up @ TANDEM IPNOrsay

cross section study will be pursued in CEA DAM Bruyèresle-Chatel by cross sections measurements of the following Lutetium isotopes: 173,174,175,176Lu. The knowledge of the complete set of cross sections will be useful in the interpretation of radiochemical activation in the inertial confinement of fusion experiments. To infer the capture probability, gammas rays are still detected in coincidence with the ejectile, which is fully identified by large efficiency position sensitive detectors. It consists of two large area $\Delta \mathrm{E}$ E telescopes placed symmetrically at $130^{\circ}$ with regard to the $3 \mathrm{He}$ beam. The E detectors are two $\mathrm{Si}(\mathrm{Li}) 3000 \mu \mathrm{m}$ detectors. The $\Delta \mathrm{E}$ detectors are two $300 \mu \mathrm{m}$ silicon position sensitive detectors, whose $\mathrm{X}-\mathrm{Y}$ strips provide the angle of the detected particle. From the signals delivered by the telescope, the transfer channel could be fully identified and kinematically reconstructed. We use a set of four C6D6 liquid scintillators for gamma detection and the total energy detection principle in combination with the pulse height weighting technique Refs $[5,6]$ to infer the total number of detected gamma. The figure 8 illustrates our experimental set-up. The same technique was used to obtain the $233 \mathrm{~Pa}(\mathrm{n}, \gamma)$ cross section, but this new set-up has a larger efficiency (ten times more) than the one used in our previous surrogate experiments Ref [7]. However, in this experiment we will considerably improve the set-up by adding several germanium detectors. In that way it will be possible to measure low-lying $\gamma$-ray transition intensities as a function of the compound nucleus excitation energy, which is an experimental way to (i) investigate the difference in spin distributions between transfer and neutron-induced reactions, and (ii) to test theoretical calculations. The detection of discrete low-lying $\gamma$-rays emitted by the residual nucleus in coincidence with the proton exit chanel reaction will be useful to infer the calculated level spin distribution and to constrain the reaction models. That requires modelling the decay of the compound nucleus and fitting the relevant parameters of the spin distribution to reproduce the experimental data. In addition, $\gamma$-ray intensity ratios can be measured. This observable is very sensitive to the spin distribution and has the advantage that it can be directly compared to the same quantity measured in a neutron-induced experiment. In order to illustrate the influence of the spin distribution on this observable we performed Hauser-Feshbach calculations of the $\gamma$ - 


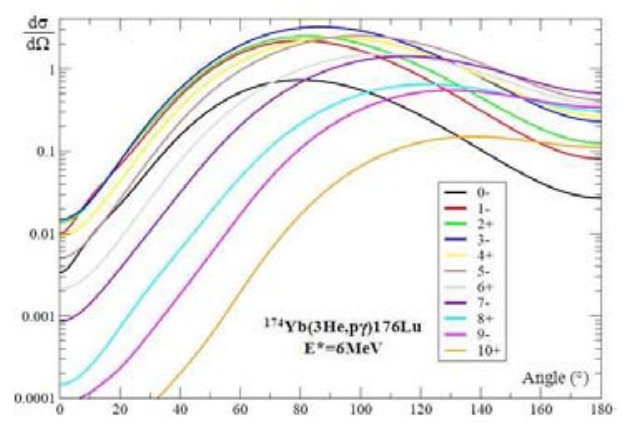

Fig. 4. Angular distribution, $\mathrm{d} \alpha / \mathrm{d} \theta$ in a.u, of the ejected proton in the $174 \mathrm{Yb}(3 \mathrm{He}, \mathrm{p} \gamma) 176 \mathrm{Lu}$ reaction for different $\mathrm{J} \pi$ states and compound nucleus excitation energy of $6 \mathrm{MeV}$.

ray intensity ratios in neutron-induced reactions using the TALYS code. In the following we will consider only the $175 \mathrm{Lu}(\mathrm{n}, \gamma)$ and the $174 \mathrm{Yb}(3 \mathrm{He}, \mathrm{p} \gamma) 176 \mathrm{Lu}$ reactions. Figure 1 shows the intensity ratios for a 6-/7- transition over a 1-/1- transition and for the same 6-/7- transition over a $6+/ 囚 4+$ transition as a function of the incident neutron energy for the $175 \mathrm{Lu}(\mathrm{n}, \gamma)$ reaction. Note that the ground state spin and parity of $175 \mathrm{Lu}$ is $7 / 2+$, so s-wave neutrons will populate spin states of $\mathrm{J} \pi=3+-4+$ in the $176 \mathrm{Lu}$ compound nucleus. Moreover, we could have a look on the isomeric ratio which are well feed for $176 \mathrm{Lu}(8+: 425 \mathrm{keV}, \mathrm{tl} / 22$ ns; $1+: 195 \mathrm{keV}, \mathrm{t} 1 / 2 \quad 35 \mathrm{~ns} ; 3-: 658 \mathrm{keV}, \mathrm{t} 1 / 2 \quad 6.3 \mathrm{~ns})$

The calculation of such quantities in a $(3 \mathrm{He}, \mathrm{p})$ reaction is a more difficult task since it requires an accurate description of the transferred angular momentum by deuteron transfer leading to unbound states and their decay. Figure 2 shows the calculated angular distribution for the formation of a $\mathrm{J} \pi$ compound nucleus state located at $6 \mathrm{MeV}$ excitation energy. The differential cross section was obtained using the DWBA FRESCO code. As seen in figure 5, the spin distribution can be modified by changing the proton detection angle.

Figure 5 shows the expected spin distribution of the compound nucleus in the transfer reaction for protons detected at $108^{\circ}, 130^{\circ}$ and $152^{\circ}$ for three excitation energies: 6,7 and $8 \mathrm{MeV}$. In the same figure, the spin distribution obtained in the $175 \mathrm{Lu}(\mathrm{n}, \gamma) 176 \mathrm{Lu}$ reaction is shown for three neutron incident energies corresponding to the same excitation energies. The latter distributions have been obtained using the TALYS code. Although broader, "surrogate" distributions present average values between 4 and $5 \hbar$ that are rather similar to those of neutron reactions. In addition, the use of germanium detectors will considerably help in the identification of the decaying nucleus. For example, with C6D6 detectors alone it is not possible to disentangle between a gamma arising from the $(3 \mathrm{He}, \mathrm{p} \gamma)$ and from the $(3 \mathrm{He}, \mathrm{pn} \gamma)$ reaction. Statistical model calculations are used to correct for this effect. However, by including germanium detectors in our set-up we can measure excitation functions for certain low-lying transitions related to the $(3 \mathrm{He}, \mathrm{pn} \gamma)$ reaction. These excitation functions represent a precious piece of information for improving the models used to correct for this effect.

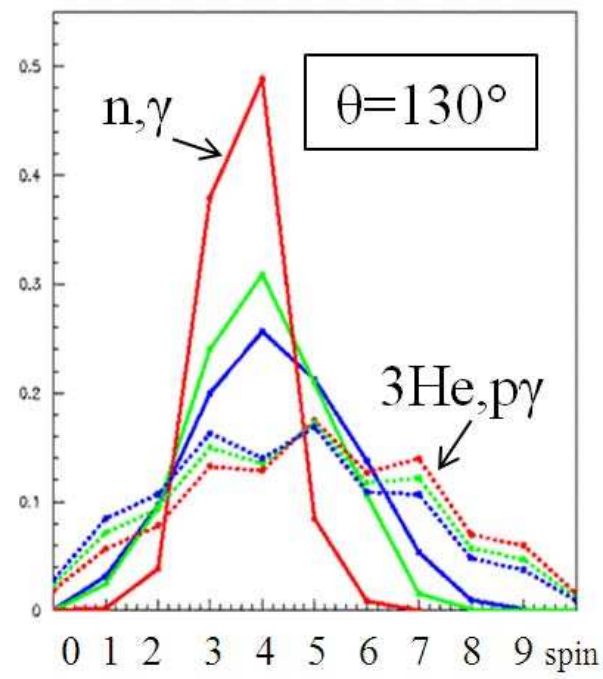

Fig. 5. Spin distribution of the compound nucleus in the $175 \mathrm{Lu}(\mathrm{n}, \gamma) 176 \mathrm{Lu}$ (solid lines) for $\mathrm{En}=100 \mathrm{keV}$ (red), $1 \mathrm{MeV}$ (green) and $2 \mathrm{MeV}$ ( blue) and in the $174 \mathrm{Yb}(3 \mathrm{He}, \mathrm{p} \gamma) 176 \mathrm{Lu}$ (dotted lines), for $\mathrm{E}^{*}=6 \mathrm{MeV}$ (red), $7 \mathrm{MeV}$ (green) and $8 \mathrm{MeV}$ ( blue).

\section{Conclusion}

This study is extremely important in view of the application of the surrogate method to infer radiative capture cross sections of minor actinides, which are crucial for nuclear waste incineration. In the future, we plan to use the $237 \mathrm{~Np}(\mathrm{n}, \gamma), 238 \mathrm{U}(\mathrm{n}, \gamma)$ and $232 \mathrm{Th}(\mathrm{n}, \gamma)$ cross sections to validate the $236 \mathrm{U}(3 \mathrm{He}, \mathrm{p} \gamma) 238 \mathrm{~Np}, 238 \mathrm{U}(\mathrm{d}, \mathrm{p} \gamma) 239 \mathrm{U}$ and $232 \mathrm{Th}(\mathrm{d}, \mathrm{p} \gamma) 233 \mathrm{Th}$ surrogate reactions respectively. Unfortunately, the latter surrogate reactions require thin metallic isotopically-pure actinide targets which are not yet available. We would like also to stress that the surrogate approach is very general and could be used to determine cross sections of a large variety of nuclei and applications.

\section{References}

1. J.D. Cramer, H.C. Britt, Nucl. Sci. and Eng. 41 (1970) 177

2. ] W. Hauser, H. Feshbach, Phys.Rev. 87 (1952) 366

3. ] V. Weisskopf and D.H. Ewing, Phys. Rev. 57, 472 (1940)

4. M. Petit et al., Nucl. Phys. A 735 (2004) 345.

5. J. N. Wilson et al., Nucl. Instr. Meth. A 511 (2003) 388

6. A. Borella et al., Nucl. Instr. Meth. A 577 (2007) 626

7. S. Boyer et al., Nucl Phys A 775 (2006) 175

8. K. Wisshak et al., Phys. Rev. C 73 (2006) 015807

9. M. Heil et al., Nucl. Instr. Meth. A 459 (2001) 229 